\title{
ORIGINAL
}

\section{TUBERCULOSIS EN LA COMUNIDAD DE MADRID. INCIDENCIA EN PERSONAS EXTRANJERAS Y ESPAÑOLAS DURANTE EL PERÍODO 1996-2004}

\author{
María Ordobás Gavín (1), Soledad Cañellas Llabrés (1), Cristina Garcia Fernández (1), Luis García \\ Comas (1), Ma Angeles Gutiérrez Rodríguez (1), Inmaculada Rodero Garduño (1), Juan García \\ Gutierrez (1), Rosa Ramirez Fernández (1), Fernándo Rodríguez Artalejo (2)
}

(1) Servicico de Epidemiología. Dirección General de Salud Pública y Alimentación de la Comunidad de Madrid.

(2) Departamento de Medicina Preventiva y Salud Pública. Facultad de Medicina. Universidad Autónoma de Madrid.

\section{RESUMEN}

Fundamento: La inmigración desde países de alta prevalencia de tuberculosis hacia otros más desarrollados afecta a la evolución global de la tuberculosis en los últimos años. El objetivo de este estudio fue describir la influencia de los casos de tuberculosis en personas extranjeras sobre la incidencia de la enfermedad en la Comunidad de Madrid en el período 1996 a 2004.

Métodos: Los datos procedían del Registro Regional de Casos de Tuberculosis, y del padrón de 1996 y los padrones continuos de 1998 a 2004. Se estimó la incidencia de tuberculosis según país de origen desde 1996 a 2004, por sexo y grupos de edad. Se calcularon $\chi^{2}$ para tendencia lineal, razones de incidencia y proporción de casos en personas extranjeras.

Resultados: La incidencia de tuberculosis pasó de 34,3 casos por $10^{5}$ habitantes en 1996 a 16,9 casos por $10^{5}$ habitantes en 2004. Para los nacidos en España cambió de 33,2 casos por $10^{5}$ habitantes en 1996 a 12,7 casos por $10^{5}$ habitantes en 2004, y para los extranjeros de 50,5 casos por $10^{5}$ habitantes en 1996 a 42,9 casos por $10^{5}$ habitantes en 2004. La razón de la incidencia entre extranjeros y españoles fue superior a 1 en todos los años, con valor máximo en 2003, en el que se detectaron 4,2 casos en extranjeros por cada caso en españoles (IC 95\% 3,7-4,7). El porcentaje de casos extranjeros pasó del 5,2\% en 1996 al 35,1\% en 2004.

Conclusiones: La incidencia de tuberculosis en extranjeros fue mayor que entre los españoles y no disminuyó significativamente en el período 1996-2004, lo que está contribuyendo a que la tuberculosis se haya estabilizado. Esta situación y las características de esta población han de ser tenidas en cuenta en los esfuerzos para el control de esta enfermedad.

Palabras Clave: Tuberculosis. Prevención de la enfermedad. Inmigración.

\section{Correspondencia:}

María Ordobás Gavín

Servicio de Epidemiología

Dirección General de Salud Pública y Alimentación de la

Comunidad de Madrid

C/ Julián Camarillo, 4B-28027 Madrid

Teléfono: 912052232. Fax: 912040173

Correo electrónico: maria.ordobas@salud.madrid.org

\section{ABSTRACT}

\section{Tuberculosis in the Autonomous Region of Madrid, Spain. Incidence among Foreign and Spanish Individuals during the 1996-2004 Period}

Backgound: Immigration from countries having a high prevalence of tuberculosis to other more highly-developed countries has a bearing on the overall evolution of tuberculosis in the latter. This study is aimed at describing the influence of the cases of tuberculosis among foreign individuals on the incidence of this disease in the Autonomous Community of Madrid during the 1996-2004 period.

Methods: The data was taken from the Regional Tuberculosis Case Registry and from the 1996 census and the continuous censuses from 1998 to 2004. The tuberculosis incidence was estimated by country of origin from 1996 to 2004, by gender and by age groups. A calculation was made of the $c 2$ for the linear trend, reasons for incidence and proportion of cases among foreign individuals.

Results: The incidence of tuberculosis went from 34.3 cases per105 inhabitants in 1996 to 16.9 cases per105 inhabitants in 2004. For those born in Spain, it changed from 33.2 cases per 105 inhabitants in 1996 to 12.7 cases per 105 inhabitants in 2004; and for foreign individuals, from 50.5 cases per 105 inhabitants in 1996 to 42.9 cases per 105 inhabitants in 2004. The reason for the incidence between foreign and Spanish individuals was greater than 1 for all years, the maximum figure having been in 2003, when 4.2 cases were detected among foreigners for every case among Spanish individuals (95\% CI 3.7-4.7). The percentage of foreign cases went from $5.2 \%$ in 1996 to $35.1 \%$ in 2004 .

Conclusions: The incidence of tuberculosis among foreigners was greater than among Spanish individuals and did not significantly decrease in the 1996-2004 period, which is contributing to tuberculosis having stabilized. This situation and the characteristics of this population must be taken into account in the efforts for controlling this disease.

Keywords: Tuberculosis. Prevention. Immigration. Epidemiology. 


\section{INTRODUCCIÓN}

Actualmente no hay ninguna duda de que la inmigración desde países de alta endemia afecta a la evolución global de la tuberculosis en los más desarrollados, sin embargo, sí hay controversia sobre algunos de los aspectos de esa influencia así como sobre las medidas específicas a adoptar para alcanzar el control ${ }^{1}$.

En los últimos años la inmigración ha tenido un crecimiento exponencial en España. En junio de 2004, había al menos 1.776 .953 extranjeros con permiso de residencia ${ }^{2}$. En la Comunidad de Madrid los datos del padrón continuo de la población ponían de manifiesto, en ese año, que el 13,9\% de la población empadronada había nacido en un país extranjero. Por países a la cabeza se situaban los Iberoamericanos y en el primer lugar Ecuador ${ }^{3}$.

A principios de los años 90 la infección por el VIH/SIDA en España condicionó que la tuberculosis reapareciera como prioridad en Salud Pública. El control de la tuberculosis se basa en la adopción de políticas sanitarias adaptadas a las características de los casos que se están produciendo. Cuando ello supone abordar factores como la inmigración representa además un desafío para las administraciones públicas. Por otra parte, aunque la población inmigrante tiene una selección positiva de su estado de salud y un riesgo bajo de presentar tuberculosis, este riesgo es mayor que el de la población receptora ya que presenta una prevalencia de infección mayor ${ }^{4,5}$ y viven a menudo en condiciones precarias.

El objetivo de este estudio es analizar la influencia de los casos de tuberculosis en personas extranjeras sobre el conjunto de casos de la Comunidad de Madrid en el período 1996 a 2004.

\section{SUJETOS Y MÉTODOS}

Se realizó un estudio descriptivo de la incidencia de tuberculosis en la Comunidad de Madrid desde 1996 a 2004 según país de nacimiento: España frente a otro país, por sexo y grupos de edad: menores de 15 años, de 15 a 64 y mayores de 64 años.

Los datos procedían del Registro Regional de Casos de Tuberculosis. Se considera caso para su inclusión en el Registro: persona con cultivo positivo a Mycobacterium tuberculosis y/o con baciloscopia positiva a la que se le indica tratamiento antituberculoso, o al paciente con tratamiento antituberculoso durante más de tres meses o menos si fallece o se le retira el tratamiento por efectos secundarios. Para cada caso se cumplimentó un cuestionario con diversas variables, por parte del personal sanitario responsable de la notificación, o bien a partir de todos los datos que se recogen en la Sección de Epidemiología del Área de Salud desde las distintas fuentes de información: declaraciones a las Enfermedades de Declaración Obligatoria, laboratorios de microbiología que realizan baciloscopia y/o cultivo y/o tipado, y/o pruebas de sensibilidad de micobacterias, el Conjunto Mínimo Básico de Datos (CMBD) de hospitalización y otras fuentes como el Registro Regional de Casos de SIDA de la Comunidad de Madrid, el Registro de Instituciones Penitenciarias, funerarias, etc.

Como denominadores se utilizaron las poblaciones del padrón de 1996 y padrón continuo de 1998 a 2004, y para 1997 la estimación media entre 1996 y 1998.

Se calculó el porcentaje de cambio en la incidencia de tuberculosis de un año respecto al anterior y la media anual para el período 1996 a 2004. Se analizaron las tendencias de las estimaciones de incidencias mediante el cálculo de la $\chi^{2}$ para tendencia lineal, la evolución de las razones entre la 
incidencia en extranjeros y en españoles y el \% de casos extranjeros. Los intervalos de confianza se han establecido a un nivel del $95 \%$.

\section{RESULTADOS}

Desde 1996 a 2004 la incidencia de tuberculosis en la Comunidad de Madrid disminuyó una media de $9,4 \%$ anual $(\mathrm{DE}=6,7 \%, \mathrm{p}<0,05)$. Por grupo de edad, entre 15 y 65 años disminuyó un $9,9 \%$ anual $(\mathrm{DE}=8,9 \%, \mathrm{p}<0,05)$ y en los mayores de 64 años un $8,0 \%$ anual $(\mathrm{DE}=7,2 \%$, $\mathrm{p}<0,05)$; en los menores de 15 años, aunque decreció un $8,7 \%$ anual $(\mathrm{DE}=28,9 \%$, $\mathrm{p}=0,22$ ), no se alcanzó la significación estadística. Al analizar el origen de los casos, para los nacidos en España la disminución fue del $12,7 \%$ anual (DE $=6,0 \%$, $\mathrm{p}<0,05)$, y para los extranjeros del $5,5 \%$ anual $(\mathrm{DE}=28,7 \%, \mathrm{p}=0,31)$ y tuvo importantes variaciones anuales (tabla 1). Para los españoles, la incidencia específica disminuyó entre los hombres una media de $15,3 \%$ anual $(\mathrm{DE}=7,5 \%, \mathrm{p}<0,05)$, y entre las mujeres del $12,4 \%$ anual $(\mathrm{DE}=6,9 \%$, $\mathrm{p}<0,05)$. En el caso de los extranjeros, para los hombres el descenso fue del 5,8\% anual ( $\mathrm{DE}=30,3 \%, \mathrm{p}=0,32)$, y para las mujeres la tendencia tampoco alcanzó la significación estadística, y se invirtió, aumentando la incidencia una media de $0,1 \%$ anual $(\mathrm{DE}=25,6 \%, \mathrm{p}=0,20)$ (figura 1). Por grupos de edad, entre los autóctonos la incidencia específica disminuyó en todos los grupos: entre los menores de 15 años una media del $11,4 \%$ anual $(\mathrm{DE}=19,4 \%, \mathrm{p}<0,05)$, para los casos entre 15 y 64 años del $15,6 \%$ anual $(\mathrm{DE}=10,5 \%$, $\mathrm{p}<0,05)$, y para los mayores de 64 años del $8,4 \%$ anual $(\mathrm{DE}=6,7 \%, \mathrm{p}<0,05)$. Para los extranjeros también decreció la inci-

Figura 1

Incidencia anual de tuberculosis global y según sexo y país de origen de los casos. Comunidad de Madrid, 1996-2004

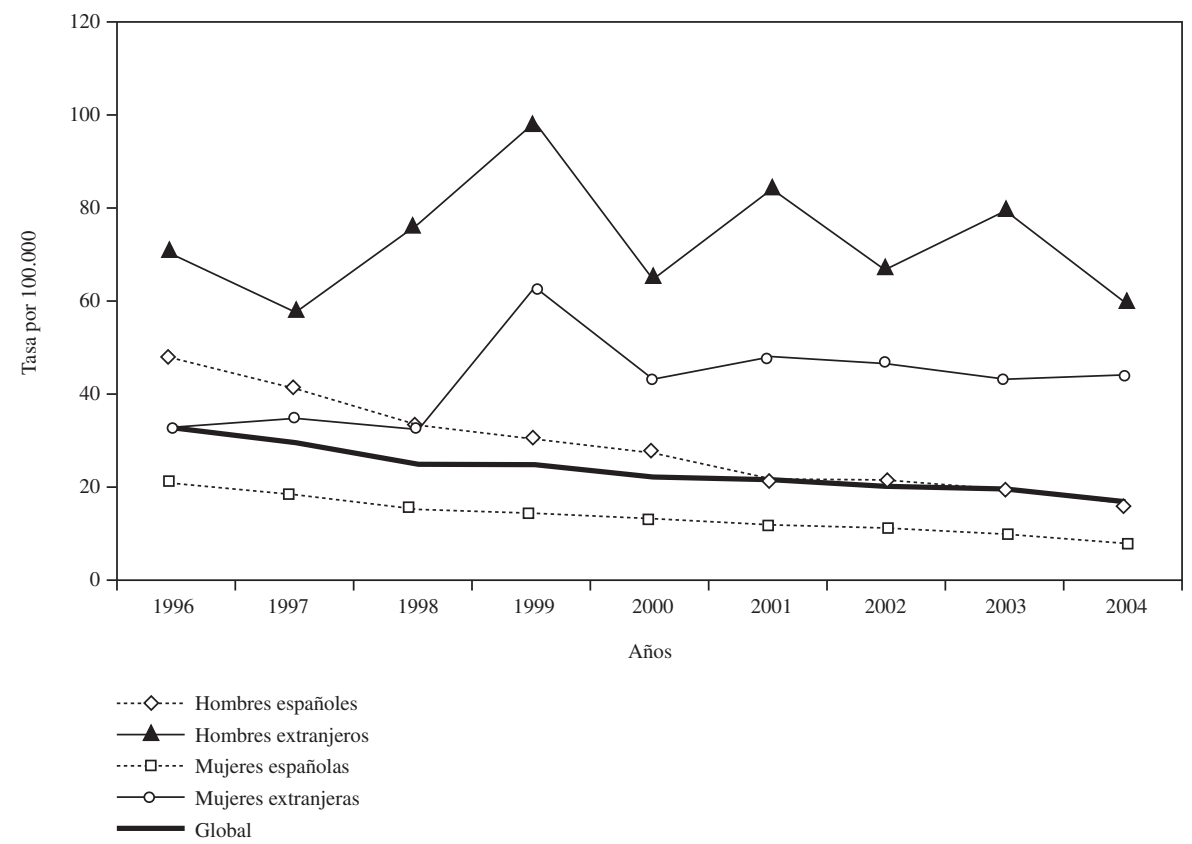


Figura 2

Incidencia anual de tuberculosis según edad y país de origen de los casos. Comunidad de Madrid, 1996-2004

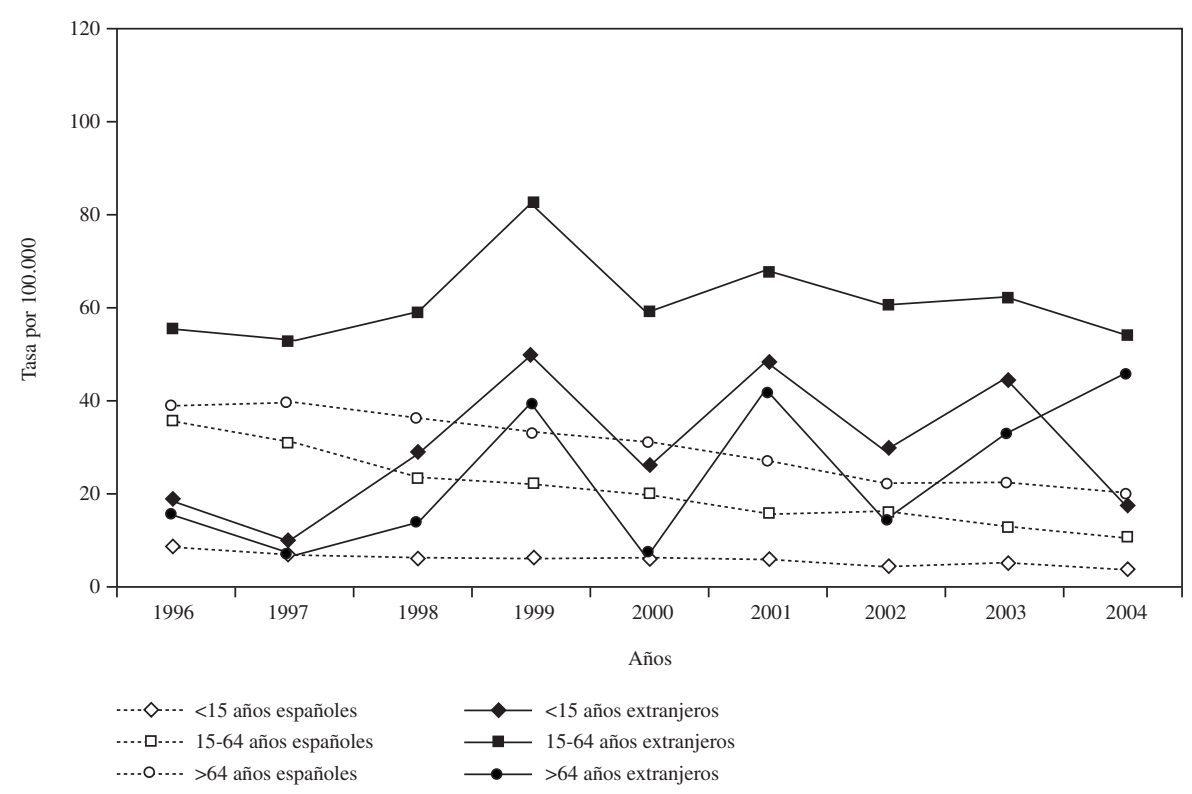

dencia específica en todos los grupos, en 2 de los 3 grupos: para los menores de pero con importantes variaciones anuales 15 años una media del $25,2 \%$ anual y sin alcanzar la significación estadística $(\mathrm{DE}=81,1 \%, \mathrm{p}=0,80)$, entre $\operatorname{los} 15$ y 64

Tabla 1

Distribución del número de casos anuales españoles y extranjeros e incidencia de turberculosis por sexo y edad. Comunidad de Madrid, 1996-2004

\begin{tabular}{|c|c|c|c|c|c|c|c|c|c|}
\hline & 1996 & 1997 & 1998 & 1999 & 2000 & 2001 & 2002 & 2003 & 2004 \\
\hline $\begin{array}{l}\text { Número de Casos } \\
\text { Total } \\
\text { Españoles } \\
\text { Extranjeros }\end{array}$ & $\begin{array}{r}1724 \\
1634 \\
90\end{array}$ & $\begin{array}{r}1518 \\
1422 \\
96\end{array}$ & $\begin{array}{r}1301 \\
1176 \\
125\end{array}$ & $\begin{array}{r}1278 \\
1102 \\
176\end{array}$ & $\begin{array}{r}1180 \\
978 \\
202\end{array}$ & $\begin{array}{r}1135 \\
831 \\
304\end{array}$ & $\begin{array}{r}1135 \\
829 \\
306\end{array}$ & $\begin{array}{r}1150 \\
727 \\
423\end{array}$ & $\begin{array}{l}982 \\
637 \\
345\end{array}$ \\
\hline $\begin{array}{l}\% \text { de Casos tuberculosis pulmonar } \\
\text { Total } \\
\text { Españoles } \\
\text { Extranjeros }\end{array}$ & $\begin{array}{l}69,9 \\
69,8 \\
71,1\end{array}$ & $\begin{array}{l}67,8 \\
67,2 \\
77,1\end{array}$ & $\begin{array}{l}67,4 \\
66,7 \\
73,6\end{array}$ & $\begin{array}{l}72,3 \\
72,0 \\
73,9\end{array}$ & $\begin{array}{l}69,1 \\
69,5 \\
66,8\end{array}$ & $\begin{array}{l}71,9 \\
72,2 \\
71,0\end{array}$ & $\begin{array}{l}72,9 \\
71,3 \\
77,1\end{array}$ & $\begin{array}{l}75,4 \\
73,2 \\
79,2\end{array}$ & $\begin{array}{l}72,7 \\
73,4 \\
71,6\end{array}$ \\
\hline $\begin{array}{l}\text { Incidencia x } 10^{5} \\
\text { Total } \\
\text { Españoles } \\
\text { Extranjeros }\end{array}$ & $\begin{array}{l}34,3 \\
33,2 \\
50,5\end{array}$ & $\begin{array}{l}30,0 \\
29,1 \\
46,1\end{array}$ & $\begin{array}{l}25,6 \\
24,2 \\
52,5\end{array}$ & $\begin{array}{l}24,8 \\
22,4 \\
79,0\end{array}$ & $\begin{array}{l}22,7 \\
20,3 \\
53,7\end{array}$ & $\begin{array}{l}21,1 \\
16,9 \\
65,6\end{array}$ & $\begin{array}{l}20,6 \\
16,6 \\
56,2\end{array}$ & $\begin{array}{l}20,1 \\
14,5 \\
60,9\end{array}$ & $\begin{array}{l}16,9 \\
12,7 \\
42,9\end{array}$ \\
\hline $\begin{array}{l}\text { Incidencia x } 10^{5} \\
\text { Hombres } \\
\text { Mujeres }\end{array}$ & $\begin{array}{l}48,9 \\
20,8\end{array}$ & $\begin{array}{l}41,7 \\
19,2\end{array}$ & $\begin{array}{l}35,8 \\
16,1\end{array}$ & $\begin{array}{l}33,5 \\
16,8\end{array}$ & $\begin{array}{l}30,3 \\
15,6\end{array}$ & $\begin{array}{l}27,8 \\
14,9\end{array}$ & $\begin{array}{l}26,6 \\
14,8\end{array}$ & $\begin{array}{l}26,6 \\
14,0\end{array}$ & $\begin{array}{l}21,4 \\
12,6\end{array}$ \\
\hline $\begin{array}{l}\text { Incidencia x } 10^{5} \\
<15 \text { años } \\
15-64 \text { años } \\
>64 \text { años }\end{array}$ & $\begin{array}{r}9,4 \\
37,3 \\
39,2\end{array}$ & $\begin{array}{r}7,4 \\
32,5 \\
39,2\end{array}$ & $\begin{array}{r}7,8 \\
26,3 \\
36,6\end{array}$ & $\begin{array}{r}8,0 \\
25,8 \\
33,5\end{array}$ & $\begin{array}{r}7,7 \\
23,7 \\
31,4\end{array}$ & $\begin{array}{r}9,4 \\
21,7 \\
28,3\end{array}$ & $\begin{array}{r}6,6 \\
22,4 \\
23,1\end{array}$ & $\begin{array}{r}9,4 \\
21,3 \\
23,2\end{array}$ & $\begin{array}{r}6,2 \\
17,9 \\
21,5\end{array}$ \\
\hline
\end{tabular}


Tabla 2

Distribución anual de la razón de incidencias de tuberculosis en extranjeros y españoles y del \% de casos extranjeros. Comunidad de Madrid, 1996-2004

\begin{tabular}{|l|c|c|c|c|c|c|c|c|c|}
\hline & $\mathbf{1 9 9 6}$ & $\mathbf{1 9 9 7}$ & $\mathbf{1 9 9 8}$ & $\mathbf{1 9 9 9}$ & $\mathbf{2 0 0 0}$ & $\mathbf{2 0 0 1}$ & $\mathbf{2 0 0 2}$ & $\mathbf{2 0 0 3}$ & $\mathbf{2 0 0 4}$ \\
\hline Riesgo Relativo* & 1,5 & 1,6 & 2,2 & 3,5 & 2,6 & 3,9 & 3,4 & 4,2 & 3,4 \\
IC al 95\% & $1,2-1,9$ & $1,3-1,9$ & $1,8-2,6$ & $3,0-4,1$ & $2,3-3,1$ & $3,4-4,4$ & $3,0-3,8$ & $3,7-4,7$ & $3,0-3,9$ \\
\hline \% de casos Global & 5,2 & 6,3 & 9,6 & 13,8 & 17,1 & 26,8 & 27,0 & 36,8 & 35,1 \\
Hombres & 4,9 & 5,4 & 9,5 & 12,1 & 15,1 & 25,9 & 24,7 & 36,6 & 33,5 \\
Mujeres & 5,9 & 7,9 & 9,8 & 16,9 & 20,7 & 28,1 & 30,8 & 37,1 & 37,1 \\
& & & & & & & & & \\
$<15$ a & 4,1 & 3,5 & 11,9 & 16,7 & 17,5 & 31,9 & 32,7 & 45,4 & 40,4 \\
15-64 a & 6,3 & 7,9 & 12,1 & 16,6 & 21,6 & 32,5 & 32,6 & 43,3 & 43,0 \\
> 64 a & 0,7 & 0,4 & 0,8 & 2,3 & 0,4 & 3,6 & 1,6 & 4,1 & 3,8 \\
\hline
\end{tabular}

* Riesgo Relativo: Incidencia extranjeros/ Incidencia autóctonos.

años del 2,1\% anual (DE=20,9\%, $\mathrm{p}=0,92)$, y para los mayores de 64 años del $74,6 \%$ anual ( $\mathrm{DE}=223,4 \%, \mathrm{p}<0,05)$ (figura 2).

En los años 2000, 2001 y 2004 la proporción de casos pulmonares fue mayor en españoles que en extranjeros, en el resto de los años la proporción de casos pulmonares fue mayor en extranjeros (tabla 1).

La razón entre la incidencia en extranjeros y en autóctonos fue superior a 1 en todos los años estudiados, con un valor máximo en 2003, en el que se detectaron 4,2 casos en extranjeros por cada caso en español (IC al 95\% entre 3,7-4,7) (tabla 2). La proporción de casos extranjeros pasó del 5,2\% en 1996 al $35,1 \%$ en 2004; por sexo la mayor variación se ha producido en mujeres y por edad en los menores de 15 años (tabla 2).

\section{DISCUSIÓN}

Los resultados del estudio indican que, pese al notable descenso de la tuberculosis en los españoles, la inmigración que se está produciendo en los últimos años en la Comunidad de Madrid, procedente de áreas geográficas con alta frecuencia de tuberculosis, está condicionando el mantenimiento de su incidencia. Esta situación debe de ser tenida en cuenta al planificar las acciones de control y garantizar el acceso a ellas de este grupo de la población.
La incidencia de tuberculosis en la Comunidad de Madrid es similar a otras zonas de España. Así, en 2004 la incidencia en Cataluña fue de 21,6 casos por $10^{5}$ habitantes, un 6,2\% menor que la del año 2003 y con una proporción de casos en inmigrantes del $28,8 \%{ }^{6}$. En la ciudad de Barcelona la incidencia en ese mismo año fue de 25,9 casos por $10^{5}$ habitantes, con un descenso respecto a 2003 del $18 \%$, y con un $35,2 \%$ de los casos nacidos fuera de España ${ }^{7}$. En Galicia, la incidencia en 2003 fue de 45,3 casos por $10^{5}$ habitantes y de ellos menos del $3 \%$ eran extranjeros ${ }^{8}$. Así pues, con cifras similares o incluso menores de incidencia, en territorios donde predominan las mayores concentraciones urbanas de población del Estado, la condición de extranjero es más frecuente entre los casos y por ello, dado el gran incremento de este grupo de la población en nuestro país, el declive de la tuberculosis se ha detenido. A su vez, los resultados de nuestro estudio son coincidentes con la tendencia observada en dos Áreas Sanitarias de la Comunidad de Madrid ${ }^{9}$. Para todo el territorio del Estado Español, en un estudio realizado entre 1996 y $1997^{10}$ sólo el $2,9 \%$ de los casos tenía nacionalidad extranjera. Por tanto, además de las diferencias territoriales se detecta un cambio en la proporción de inmigrantes, que se ha producido en muy poco espacio de tiempo.

Nuestros resultados son consecuencia, principalmente, de que la incidencia en las 
zonas de procedencia de nuestra inmigración es muy alta, entre las mayores del mundo: así, en Ecuador se registran entre 100-300 casos por $10^{5}$ habitantes al año ${ }^{11}, \mathrm{y}$ las personas inmigrantes han tenido en su niñez y adolescencia un riesgo alto de infección ${ }^{5}$, a lo que se une que en el país receptor están expuestos a factores como déficits en la alimentación o estrés, que aumentan la vulnerabilidad a la enfermedad por su efecto sobre la situación general e inmunológica, principalmente en los primeros meses y años de su llegada, en los que son corrientes niveles altos de precariedad económica y social. En países desarrollados la edad de presentación de los casos tiende a desplazarse hacia edades más tardías, reflejando tasas de infección elevadas en el pasado $^{12}$, sin embargo el cambio demográfico ha condicionado el patrón obtenido, en el que la tendencia se modifica entre los grupos de edad más jóvenes, en los que el porcentaje de extranjeros es alto. El aumento progresivo en la razón entre la incidencia en extranjeros y autóctonos también se observa en otros países desarrollados ${ }^{13}$, derivado de la mayor disminución de casos entre la población autóctona que entre la extranjera.

Los estudios de biología molecular permiten valorar de forma específica el grado de transmisión entre población autóctona e inmigrante y parece que en los primeros años analizados en el estudio era bajo ${ }^{14,15}$, sin embargo la situación puede estar cambiando $^{16}$. En zonas con mayor experiencia en recepción de inmigración el riesgo de infección reciente a partir de casos procedentes de países de alta endemia es variable. En Holanda se ha encontrado que el $17 \%$ de los casos nacidos en Holanda estaban infectados por cepas procedentes de pacientes extranjeros ${ }^{17}$, en cambio en Dinamarca las cifras son mucho menores ${ }^{18}$.

En los países industrializados la incidencia de tuberculosis a partir de los 15 años es más elevada en hombres que en muje- res ${ }^{19,20}$, situación también encontrada en nuestro estudio. Sin embargo, en el futuro esto puede variar en virtud de la población inmigrante. Nuestros resultados muestran que la tendencia en las mujeres tiene una pendiente más suave, y en el caso de las extranjeras es la única serie que ha mostrado tendencia ascendente.

Los aspectos básicos del control de la tuberculosis en inmigrantes no difieren de los de la población autóctona: diagnóstico precoz y tratamiento completo hasta la curación $^{21}$. A estos dos pilares del control se une el tratamiento de la infección latente entre los contactos recién infectados ${ }^{22}$. La incidencia de casos puede reducirse, en ausencia de VIH/SIDA, hasta un 10-25\% anual $^{23}$. Para conseguirlo, en nuestro medio es necesario garantizar unas prácticas asistenciales adecuadas, incluyendo el tratamiento directamente supervisado en algunos casos y medidas preventivas eficaces, tanto sobre la población inmigrante como sobre la autóctona. En el caso de los inmigrantes se requieren medidas específicas que permitan el acceso inmediato a la asistencia sanitaria. En España, en un estudio retrospectivo en el que se analizó el desenlace del tratamiento antituberculoso en casos diagnosticados entre mayo de 1996 y abril de 1997, se encontró que el origen extranjero estaba asociado a resultados potencialmente insatisfactorios, que incluían el fracaso terapéutico, el traslado e interrupción del tratamiento ${ }^{24}$.

En un estudio sobre población inmigrante en Canadá encontraron que los factores de riesgo asociados a la enfermedad eran la sospecha diagnóstica en el reconocimiento médico a la entrada al país y venir de un país con incidencia superior a 100 casos por $10^{5}$ habitantes/año; a continuación la mayor edad y la menor clase social, medida a través del nivel educativo ${ }^{25}$.

La indicación de los cribados tuberculínicos en los inmigrantes en el primer contacto 
con el sistema sanitario a su llegada, como se recomienda en diversos protocolos ${ }^{26}$, puede ser más discutible. Su objetivo es el tratamiento de la infección latente. Sin embargo, la prueba de la tuberculina no permite diferenciar que la infección latente sea una infección reciente. Este inconveniente, unido a otros, como las dificultades para el cumplimiento del tratamiento, analizados junto con las ventajas que producirían, hacen concluir que no está indicada la búsqueda activa de infección ni la quimioprofilaxis en inmigrantes que no presenten síntomas ni tengan otro factor de riesgo de tener tuberculosis ${ }^{1}$.

Si bien los datos procedentes de la Vigilancia Epidemiológica pueden estar limitados por el subregistro, el Registro Regional de Casos de Tuberculosis de la Comunidad de Madrid se ha evaluado de forma parcial en varias ocasiones, y los resultados han concluido que la recogida de datos de distintas fuentes de información y el cruce periódico con otros registros consiguen una exhaustividad alta ${ }^{27-29}$. Las fuentes de información del Registro se han mantenido constantes durante el período analizado.

La principal limitación del estudio que hay que tener en consideración es la posible sobreestimación de la incidencia en extranjeros, sobre todo en los primeros años analizados, ya que las estadísticas oficiales de la población pueden no haber recogido a todos, ya que una parte estaba sin regularizar ${ }^{30}$.

En conclusión, la incidencia de tuberculosis en la Comunidad de Madrid presenta una tendencia descendente, de mayor magnitud en hombres que en mujeres, y más entre los adultos y jóvenes que entre los mayores de 65 años. Esta tendencia descendente es mayor en todos los grupos al considerar sólo los casos nacidos en España, mientras que entre los extranjeros la incidencia presenta mucha variabilidad interanual. La llegada de población inmigrante está contribuyendo a que la incidencia de tuberculosis se haya estabilizado.

\section{AGRADECIMIENTOS}

A todos los profesionales implicados en la Red de Vigilancia Epidemiológica de la Comunidad de Madrid: facultativos y otros profesionales sanitarios que detectan y notifican los casos, y a los Epidemiólogos de las Áreas de Salud, ya que sin su trabajo continuado no hubiera sido posible realizar este estudio.

\section{BIBLIOGRAFÍA}

1. Sanz-Peláez O, Caminero-Luna JA, Pérez-Arellano JL. Tuberculosis e inmigración en España. Evidencias y controversias. Med Clin (Barc). 2006; 126(7):259-69.

2. Secretaria de Estado de inmigración y emigración. Anuario estadístico de inmigración 2004. Disponible en: http: //extranjeros.mtas.es/es/general/ANUARIO_INMIGRACION _2004.PDF (a 15 de febrero de 2007)

3. Instituto de Estadística de la Comunidad de Madrid. Datos detallados 2004. Disponible en: http://www.madrid.org/iestadis/fijas/estructu/dem ograficas/padron/estructupc.htm (a 15 de febrero de 2007)

4. Pérez Arellano JL, Hernández García A, Sanz Peláez O, Angel-Moreno Maroto A. Inmigración africana en Canarias e infección tuberculosa. Med Clin (Barc). 2002;118:37-8.

5. Lacalle Rodríguez-Labajo M, Gil Juberías, Sagardui Villamor JK, González López E, Martínez Ruíz R, Orden Martínez B. Resultados de la aplicación de un examen de salud en la población inmigrante. Aten Primaria. 2000; 25:634-8.

6. Programa de Prevenció i Control de la Tuberculosi de la Direcció General de Salut Pública de la Generalitat de Catalunya. La tuberculosi a Catalunya 1'any 2004. Butlletí Epidemiologic de Catalunya. 2006;23:145-9.

7. Programa de Prevenció i Control de la Tuberculosi de Barcelona. La tuberculosi a Barcelona. Barcelona: Agència de Salut Pública del Ajuntament de Barcelona; 2006.

8. Fernández Nogueira E, Cruz Ferro E. 1996-2005: 10 años del programa gallego de prevención y control de la tuberculosis ¿dónde estamos?. Pneuma. 2006;5:81-84. 
9. Íñigo J, Arce A, Rodríguez E, García de Viedma D, Palenque E, Ruiz Serrano MJ, Cabello L, Chaves F. Tuberculosis trends in Madrid, 19942003:impact of immigration and HIV infection. Int J Tuberc Lung Dis. 2006;10:1-4.

10. Grupo de Trabajo del PMIT. La tuberculosis en España: resultados del Proyecto Multicéntrico de Investigación sobre Tuberculosis (PMIT). Ed: Instituto de Salud Carlos III. Madrid 1999.

11. WHO. Global Health Atlas. Disponible en: http://www.who.int/GlobalAtlas/predefinedReports/TB/PDF_Files/ecu.pdf (citado el 15 de febrero de 2007)

12. Chin J. El control de las enfermedades transmisibles. Washington: Organización Panamericana de la Salud; 2001.

13. Pratt R, Robison V, Navín T. Trends in tuberculosis incidence- United States, 2006.MMWR. 2007;56:245-50.

14. Íñigo Martínez J, Arce Arnáez A, Chaves Sanchez F, Palenque Mataiz E, Burgoa Arenales M. Patrones de transmisión de la tuberculosis en un área sanitaria de Madrid. Rev Esp Salud Pública. 2003;77:541-51.

15. Solsona J, Caylà J, Verdú E, Estrada MP, García S, Roca D, et al. Molecular and conventional epidemiology of tuberculosis in an inner city district. Int J Tuberc Lung Dis. 2001;5:724-31.

16. Íñigo J, García de Viedma D, Arce A, Palenque E, Alonso Rodríguez N, Rodríguez E et al. Analysis of Changes in Recent Tuberculosis Transmisión Patterns alter a Sharp Increase in Immigration. J Clin Microbiol. 2007;45:63-69.

17. Borgdorff M, Nagelkerke N, van Soolingen D, de Haas PE, Veen J, van Embden JD. Analysis of tuberculosis transmission between nationalities in the Netherlands in the period 1993-1995 using DNA fingerprinting. Am J Epidemiol. 1998;147:187-95.

18. Llillebaek T, Andersen AB, Bauer J, Dirksen A, Glismann S, de Haas P, Kok-Jensen A. Risk of Mycobacterium tuberculosis transmission in a lowincidence country due to inmigration from highincidence areas. J Clin Microbiol. 2001;39:855-61.

19. Holmes CB, Hausler H, Nunn P. Une revue des différences dans l'épidémiologie de la tuberculose selon sexe. Int J Tuberc Lung Dis. 1998;2:96-104

20. Uplekar M, Rangan S., Weiss MG, Ogden J. Attention to gender issues in tuberculosis control. Int J Tuberc Lung Dis. 2001;5:1-5
21. WHO Tuberculosis Programme: Framework for Effective Tuberculosis Control. Geneva: WHO; 1994.

22. Vidal R, Caylá J, Gallardo J, Lobo A, Martín C, Ordobás M, et al. Recomendaciones SEPAR. Normativa sobre la prevención de la tuberculosis. Arch Bronconeumol. 2002;38:441-51.

23. Frieden T. Can tuberculosis be controlled?. I J Epidemiol 2002;31:894-899.

24. Díez Ruiz- Navarro M, Hernández Espinosa JA, Díaz Franco A, Bleda Hernández MJ, Castells Carrillo C, Domínguez García A et al. Desenlace del tratamiento antituberculoso en España: resultados del Proyecto Multicéntrico de Investigación sobre Tuberculosis-2 (PMIT-2). Madrid: Instituto de Salud Carlos III; 2005.

25. Wobaser WL, Yuan L, Naus M, Corey P, Edelson J, Heywood N, Holness DL. Expanding the epidemiologic profile: risk factors for active tuberculosis in people immigrating to Ontario. Can Med Assoc J. 2000;163:823-8.

26. Grupo de trabajo de los talleres de 2001 y 2002 de la Unidad de Investigación de tuberculosis de Barcelona. Prevención y control de las tuberculosis importadas. Med Clin (Barc). 2003; 121:549-62.

27. Fernández S, Ordobás M, Hernando M, Fernández de la Hoz K, García M. Vigilancia Epidemiológica de la Tuberculosis de la Comunidad de Madrid. Exhaustividad del Registro en los Casos con Antecedentes de Estancia en Prisión. XVI Reunión Científica de la Sociedad Española de Epidemiología. Sevilla, Octubre de 1998. Rev Esp Salud Pública. 1998;72 (Supl):57-172.

28. Mayo E, Ariñez MC, Ordobás M, Iniesta D. Usefulness of tanatos as a source of recent mortality data for the epidemiologic surveillance of tuberculosis in the Community of Madrid. XXI Reunión Científica de la Sociedad Española de Epidemiología. Toledo, Octubre de 2003. Gac Sanit. 2003;17(Supl):51-198.

29. Rodríguez G, Jiménez M, Daza R, García L, Domínguez MJ, Martinez F et al. Evaluación de la Vigilancia de la tuberculosis respiratoria en el Área 6 de la Comunidad de Madrid. XXI Reunión Científica de la Sociedad Española de Epidemiología. Toledo, Octubre de 2003. Gac Sanit. 2003;17(Supl):51-198.

30. Observatorio permanente de la inmigración. Boletín del Imserso 1998. 